EUROPEAN ORGANISATION FOR NUCLEAR RESEARCH

CERN-PPE/94-181

14 November 1994

\title{
THE BEAM OBSERVATION SYSTEM OF THE ISOLDE FACILITY
}

\author{
G. J. Focker, F. Hoekemeijer, O.C. Jonsson, E. Kugler, H. L. Ravn \\ CERN, Geneva, Switzerland
}

\begin{abstract}
During the reconstruction of the ISOLDE on-line mass separator facility at CERN one of the goals was the development and implementation of an efficient and user friendly beam observation system. The pick-up devices used on the two mass separators and the beam lines are mainly Faraday cups, wire scanners and wire grids. Most of these items were specifically designed at ISOLDE, in particular, a linearly moving wire scanner. The controls, data handling and display are all integrated in the PC based control system and can be accessed under Windows like any other element of the entire machine. The strong points of this measuring system as well as the limitations of beam observation in a low energy radioactive beam facility are discussed.
\end{abstract}

(ISOLDE - General)

Presented at the Sixth Beam Instrumentation Workshop

Vancouver, Canada, 2-6 October 1994

To be published in the AIP Conference Proceedings 
1. 


\section{Introduction}

The ISOLDE on-line mass separator facility at CERN [1] is mainly used for the production of pure samples of short-lived radioactive isotopes. It consists of two electromagnetic isotope separators, the General Purpose Separator and the High Resolution Separator, the latter now in its final phase of installation. The radioactive nuclides are produced in a suitable target bombarded with the high intensity beam of $1 \mathrm{GeV}$ protons from the PS-Booster. After ionisation and acceleration to $60 \mathrm{keV}$, the ions are mass analysed. The selected beam from either of the two separators is then fed into a complex beam transport system and steered to the experiments.

The aim of the beam instrumentation is to obtain qualitative and quantitative information on intensity, distribution and position of these low intensity $(<10 \mu \mathrm{A})$ d.c. ion beams all along the beam path. In many positions the insertion of probes into the beam is impossible due to, for example, electrical potentials (as in the extraction gap) or difficult due to mechanical obstacles (as inside a magnet). In the design of the ISOLDE mass separators attention was paid to provide access for beam observation at the most critical regions such as the entrance to the magnets and the different focal points. However, if one wants to introduce several probes and a slit in a given position the available space along the beam axis may be limited, in particular if the focusing is strong. Therefore they need to be compact in the direction of the beam. In addition, emphasis was put on the probes to be easily interchangeable and radiation resistant because of the radioactive environment in which they have to operate.

The beam detection is in all cases based on the deposition of electrical charge from the beam on a probe. Unless suppressed, the emission of secondary electrons enhances the measured current by a factor depending on the surface conditions and the species and charge of ions collected.

\section{Probes}

\section{1. $\quad$ Moving wire scanners}

The pick-up needle is mounted on a small chariot which also carries the pre-amplifier. It is guided on a rail and driven back and forth by a stepping motor via a $0.1 \mathrm{~mm}$ thick piano wire. There is only one position-reference and only one direction is used for datataking to avoid the effect of mechanical hysteresis. The development of this device was started more than 10 years ago by G. Sidenius and A. Lindahl at the Niels Bohr Institute in Copenhagen [2] and since then it has been further improved at CERN.

In its standard version this beam scanner has a working range of $97 \mathrm{~mm}$ and is equipped either with a single needle of dia. $0.5 \mathrm{~mm}$ for scanning in one dimension or with a V-shaped pick-up bought from Danfysik. The former type is installed horizontally in the focal plane of the isotope separators to visualise the mass spectrum. The scanning range of this unit is limited to $3.5 \%$ of the mass spectrum. In order to cover the whole accessible mass range in the focal plane chamber of the General Purpose Separator, i.e. $13 \%$ on either side of the central mass, the scanner can be moved mechanically along the focal plane. Two scanners are mounted on the same support as backup for each other. A new version of this scanner unit with double stroke length is being developed. 
In the configuration with a $\mathrm{V}$-shaped pick-up (angle of $90^{\circ}$ ) the scanner chariot moves at $45^{\circ}$ with respect to the horizontal plane thus producing both an $\mathrm{x}$ - and a y-scan of the beam profile. Mounted on a flange together with a Faraday cup they form the standard beam observation unit as shown in figure 1. Fifteen of these units are used in all the beamlines.

Fig.1. The standard beam observation unit.

\subsection{Wire grids}

These robust devices are used where high spatial resolution is not required and where the main objectives are reliability and absence of semi-conductors because of high radiation levels near the target. Different versions with wire spacing ranging from 1,25 to $2,50 \mathrm{~mm}$ that cover a surface of $75 \times 75 \mathrm{~mm}$ are used. Some of the grids were produced at the Niels Bohr Institute, others were bought from industry.

A special model was constructed at ISOLDE with only 20 horizontal wires $(1 \mathrm{~mm}$ spacing) with the aim of measuring the vertical profile of the central beam in the focal plane of the mass separators. This unit is fitted to the slit mechanism and can be moved into the beam together with one of the defining slits.

\subsection{Faraday cups}

The standard Faraday cup is of simple construction and has a diameter of $26 \mathrm{~mm}$. No additional cooling is used as the maximum beam current does not exceed $20 \mu \mathrm{A}$ at $60 \mathrm{keV}$. Suppression of secondary electrons is achieved by applying a negative bias voltage of $130 \mathrm{~V}$ to a repeller electrode inside the cup. The background current in the system is of the 
order of $10^{-12} \mathrm{~A}$. Pneumatic drives are used for moving the cups in and out of the measuring position. These two positions are monitored by micro-switches.

In the "front ends" of the separators a special version of Faraday cup (active area $125 \mathrm{~mm}$ dia.) is installed in the short gap between the extraction electrode and the first quadrupole lens This allows to measure the total ion beam current which is accelerated out from the ion source.

\subsection{Complementary equipment}

- Slits and apertures with well defined positions and dimensions, though being passive elements, are used at ISOLDE for defining sizes and positions of beams, in particular in the focal points of the ion optical system.

- Since the main purpose of the ISOLDE facility is the production of radioactive ions a dedicated tape transport is routinely used in particular for the identification of a selected isotopic beam and, combined with radiation detectors, for optimisation of proton beam, target and ion source parameters.

- In rare cases the following setups have been employed:

i. high speed amplifiers for Faraday cup readings [3] when the ion production is

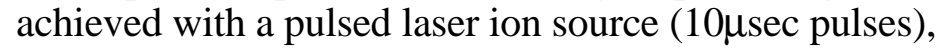

ii. a very thin $(0.05 \mathrm{~mm}$ diam.) fixed wire and electrostatic sweeping of the beam in the ISOLDE-3 high resolution separator.

\section{Drivers and amplifiers}


Fig.2. Block-diagram of the scanner controller system.

The block diagram of the scanner controller system is shown in figure 2 . The maximum electrical input current is $20 \mu \mathrm{A}$ full scale, the lowest current range actually used is $25 \mathrm{pA}$. For high sensitivity both the bandwidth of the amplifier and the scanning speed are reduced. An automatic offset regulation is done which also compensates for parts of the influence of radioactive contamination left on the needle.

Linear micro-stepping motor-drivers are used. They don't produce the continuous electro-magnetic noise caused by chopping amplifiers and provide a very smooth movement even at lower speeds.

The scanner controller-system connects to the corresponding Front End Computer (FEC) via a special PC-card. The present card is based on an industrial board which has been thoroughly modified but it has technical limitations that cause the software to be slow and not reliable enough. A new PC-card is under development. For each control channel it will have an ADC and a FIFO-register (First In First Out). It will also have an improved digital interface.

The wire grid electronics is a compact unit which is mounted as close to the wire grid as radiation levels permit. It is designed to fit to the ISOLDE control system and memorises the data which can be read at any time via a relatively long cable and an interface-card inside the FEC.

The output from the Faraday cups is measured directly by a Keithley model 617 programmable electrometer. The switching between different cups is done by two Keithley model 706 multiplexers. A IEEE-488 controller, residing in the FEC, is in charge of the hardware-software communication with the ISOLDE control system.

\section{Controls and display}


Fig.3. A focal plane scanner window showing the isotopic spectrum of radioactive Francium (mass numbers 220-227) as produced in a $\mathrm{ThC}_{2}$ target. The intensity of the ${ }^{226} \mathrm{Fr}$ beam is $0.7 \mathrm{nA}$.

The most important feature of the ISOLDE instrumentation is its complete integration in the ISOLDE control system [4]. It can be treated as any other part of the machine such as lenses or valves. All information is easily accessible even for a less skilled operator.

The ISOLDE control system is entirely set up with PCs which are all connected to the CERN-wide Ethernet (Novell software). Most of the PCs are configured as FECs interfacing with the hardware and a few are configured as consoles. The FECs run Cwritten programs under DOS. The keyboard access to a FEC is in "NODAL", a widely used interpreter language at CERN. The consoles form the user interface via Microsoft Windows 3.1 and are programmed in C and Visual Basic. Any 386 PC or better on the $\mathrm{CERN}$-wide office network may be used as a console. The database and other system-files are stored in a dedicated server.

The software in the FEC for the scanners may be complicated in comparison to that for a power supply, but for the consoles it makes very little difference whether they read data from a power supply or from a scanner. The only difference is that the approximately 270 datapoints from each scan are displayed graphically. The actual time for data handling and updating of the display is about 2 seconds. The new PC-interface will speed up data handling and allow to run four scanners simultaneously. Although the mechanical parts of the scanner would permit more than two cycles per second the updating frequency will be maintained in order to reduce the wear of critical components. For the same reason the scanners are switched off automatically after 10 minutes. Experience shows that higher 
updating speed is not really essential for setting up the beam. The spatial resolution depends on the number of datapoints and, of course, on the thickness of the pick-up needle (the apparent thickness may be higher due to possible mechanical vibrations). Figures 3 and 4 show typical scanner windows.

Fig.4. A typical beamline scanner window, ion current 9.3nA.

The readout of the wire grids with the ISOLDE control system is almost identical to that of the scanners. Although not as many datapoints are processed, the graphical display shows a fairly smooth picture similar to that of the scanners.

A window for a Faraday cup displays the measured current and the control buttons.

The Faraday cup reading of the ion current through the narrow slit in the focal plane of the separators, together with sweeping the field of the analysing magnet, is used to perform automatic mass scans. A program in the console allows to collect up to 3000 readings over the entire mass spectrum from mass 1 to 300 .

\section{Sensitivity}

Although experiments at on-line isotope separators are occasionally done with ion beam intensities as low as $1 \mathrm{ion} / \mathrm{sec}$, i.e. $10^{-19} \mathrm{~A}[5]$, it does not make sense to push the sensitivity of the beam observation equipment too far. In fact a continuous electrical background may be produced by the decay of radioactive nuclei which are implanted in the device itself or on nearby surfaces. This unwanted though decaying current, which may be 
as high as $10^{-9} \mathrm{~A}$, depends on the preceding production of radioactive beams. In the readout electronics of the scanners this effect is partly compensated by the offset regulation, but in general it sets a lower limit of some $10^{-11} \mathrm{~A}$ to the sensitivity of the beam observation for routine operation of an on-line mass separator facility.

To overcome this "blindness" when handling beams smaller than $10^{8}$ ions/sec we rely on a "mass calculator" program in the control system which, after calibration against an observable mass beam, calculates and adjusts the magnetic field (for a given acceleration voltage) for the selected mass. Thanks to the very high stability of the acceleration voltage and the excellent regulation of the magnetic field this program works accurately to $0.2 \mathrm{amu}$ within the entire mass range.

\section{Acknowledgements}

The authors thank the technical staff at ISOLDE for their meticulous work in the assembly of the different probes and the CERN ECP-Division for the preparation of the many circuit boards. Jean-Luc Dautriat, French "cooperant" in 1988/89, made valuable contributions to the development of the scanners. Our thanks go to the ISOLDE Collaboration for helping with the production of some mechanical parts at university institutes. In particular we should mention the Isotope Separator Group at the Niels Bohr Institute in Copenhagen where the scanner development was started, but interrupted in 1985 when the group was dissolved.

7. 


\section{References}

[1] E. Kugler, D. Fiander, B. Jonson, A. Przewloka, H. L. Ravn, D. J. Simon, K. Zimmer and the ISOLDE Collaboration, "The new CERN-ISOLDE on-line massseparator facility at the PS-Booster," Nucl. Instr. and Meth. B70, 41-49 (1992).

[2] Private communication.

[3] V. I. Mishin, V. N. Fedoseyev, H. J. Kluge, V. S. Letokhov, H. L. Ravn, F. Scheerer, Y. Shirakabe, S. Sundell, O. Tengblad, "Chemically selective laser ion-source for the CERN-ISOLDE on-line mass-separator facility," Nucl. Instr. and Meth. B73, 550560 (1993).

[4] C. Jonsson, O. Borch, A. Bret, R. Catherall, I. Deloose, G. J. Focker, D. Forkel, E. Kugler, G. Olesen, A. Pace, H. L. Ravn, C. Richard-Serre, G. Shering, O. Tengblad, H. J. Torgersen and the ISOLDE Collaboration, "The control system of the CERNISOLDE on-line mass-separator facility," Nucl. Instr. and Meth. B70, 541-545 (1992).

[5] J. G. Borge, H. Gabelmann, L. Johannsen, B. Jonson, G. Nyman, K. Riisager, O. Tengblad, and the ISOLDE Collaboration, "The decay of ${ }^{31}$ Ar," Nucl. Physics A515, 21-30 (1990). 